\title{
Human embryonic stem cell-derived mesenchymal stem cells have potential of high differentiation into hepatocyte-like cells through increased level of hepatocyte growth factor
}

Ji-Seon Lee ( $\sim$ jiseon80@gmail.com )

Burn Institute https://orcid.org/0000-0003-2702-4646

Dajeong Yoon

Burn Institute

Dogeon Yoon

Burn Institute

Changhwan Yeo

Burn Institute

Wook Chun ( $\square$ chun0414@hallym.ac.kr)

Hallym University Sacred Heart Hospital

\section{Research}

Keywords: human embryonic stem cell-derived mesenchymal stem cells, hepatocyte growth factor, hepatocyte, differentiation, liver failure, stem cell therapy

Posted Date: August 17th, 2020

DOI: https://doi.org/10.21203/rs.3.rs-54125/v1

License: (c) (i) This work is licensed under a Creative Commons Attribution 4.0 International License.

Read Full License 


\section{Abstract}

Background: As the aging society progresses, more patients are waiting for liver transplantation. However, the function of existing bio artificial liver is insufficient. Recently, research has been focused on stem cells to regenerate damaged liver. Most of all, the obstacle to the commercialization of widely used human adipose tissue-derived stem cells (hASCs) therapy is the limited supply of cells with consistent quality although most cell source of current stem cell therapy is hASCs. In this study, we attempt to differentiate human embryonic stem cell-derived mesenchymal stem cells (hESC-MSCs) into hepatocyte-like cells and tested whether hESC-MSCs have a potential of liver regeneration as a source of cell therapy, considering that they have characteristics of high differentiation rates, unlimited proliferation possibility from a single colony, and homogenicity.

Methods: hESC-MSCs and hASCs were cultured under condition of hepatogenic differentiation in vitro. And then various experiments including quantitative real-time PCR, immunoblotting, ELISA, immunofluorescence, Periodic acid-Schiff staining and flow cytometry analysis were performed to evaluate expression level of HGF and the extent of differentiation into hepatocyte-like cells.

Results: We found that hepatocyte growth factor (HGF), important for maintaining hepatocyte activity, is highly expressed in hESC-MSCs. Besides, it was confirmed that the expression of ALB, TDO2, and CYP2E1, which are the hepatocyte-specific genes when hepatogenic differentiation was performed under conditions even without HGF in hESC-MSCs, was higher than that when hASCs was differentiated into hepatocytes. Likewise, we concluded that hESC-MSCs could be well-differentiated into hepatocyte-like cells compared to hASCs through staining data.

Conclusions: We propose that hESC-MSCs have a promising potential as a commercial cell therapy source to treat liver failure in view of the limited available cell sources for stem cell therapy.

\section{Introduction}

As human lifespan increases, the number of patients with liver failure has been increasing. But until now, there is no cure except liver transplantation. Recently, human mesenchymal stem cells (hMSCs) therapy has been extensively studied to treat liver damage and help liver regeneration. hMSCs have differentiation potentials into ectoderm lineage cells (ex. neural cell) and endoderm lineage cell (ex. hepatocytes) as well as mesoderm lineage cells (ex. adipocytes, osteocytes, chondrocytes) $(1,2)$. Besides, hMSCs are characterized by easy isolation and expansion, safety, homing effect, immunomodulatory function, absence of ethical issue $(2,3)$. Therefore, hMSCs were widely used of source of cell therapy in the field of regenerative medicine $(2,4,5)$. However, hMSCs have limited cell numbers and replicative lifespan, and different differentiation potential dependent on individual $(3,6)$.

In while, hMSCs were manipulated by specific genes to maximize their therapeutic efficacy. Especially, it was known that MSCs overexpressing hepatocyte growth factor (HGF) were more effective to treat myocardial infarction (7), bladder fibrosis by bladder outlet obstruction (8) and ischemia reperfusion- 
induced acute lung injury (9), compared to non-treated MSCs. Actually, HGF, initially identified as a potent mitogen for hepatocytes, plays an essential role as a potent anti-fibrotic factor that inhibit tissue fibrosis in various organs, including liver, lung and kidney (10-13). Besides, there is also the following report that HGF/c-Met signaling pathway ais critically involved in MSC-induced liver repair.

Recently, research on human embryonic stem cell-derived mesenchymal stem cells (hESC-MSCs) has been actively conducted to compensate for the shortcoming of hMSCs as a cell source of stem cell therapy $(14,15)$. They express typical MSC surface markers such as CD29, CD44, and CD90 and can differentiate into mesenchymal cells including adipocytes, osteocytes, and chondrocytes $(16,17)$. These cells have also been proven to be safe for therapeutic application and show therapeutic benefits in regenerative medicine $(14,17,18)$. In other words, hESC-MSCs not only have all the advantages of hMSCs but also can produce unlimited amounts of early passage hMSCs with the consistent quality (16). In this study, we aimed to determine how much hES-MSCs express HGF and investigate whether hESCMSCs have the differentiation potential into hepatocyte-like cells.

\section{Materials And Method}

\subsection{Cell maintenance and hepatogenic differentiation in vitro}

hESC-MSCs, kindly provided by Eun Ju Lee (Seoul National University Hospital, Republic of Korea), were cultured in endothelial cell basal medium MV2 (C-22221, Promocell, Heidelberg, Germany). hASCs, provided by Anterogen Co., Ltd. (Seoul, South Korea), and hBM-MSCs (PT-2501; Lonza, Basel, Switzerland, http://www.lonza.com) were cultured in low-glucose Dulbecco's modified Eagle's medium (DMEM; cat. no.: SH30021.01; Hyclone, Logan, UT, USA) containing $0.1 \%$ gentamicin and $10 \%$ fetal bovine serum (FBS). hESC-MSCs $\left(1.3 \times 10^{6}\right.$ cells $)$ and hASCs $\left(1 \times 10^{6}\right.$ cells $)$ were seeded on fibronectin (F1141-2MG; Sigma)-coated $100-\mathrm{mm}$ tissue culture plates. The next day, the cells were washed with HBSS twice, and the medium was changed to low-glucose DMEM (in case of hASCs) or endothelial cell basal medium MV2 (in case of hESC-MSCs) containing $0.1 \%$ gentamicin, $20 \mathrm{ng} / \mathrm{mL}$ epidermal growth factor (EGF; CYT217; ProSpec, East Brunswick, NJ, USA), and 10 ng/mL bFGF (CYT-218; ProSpec) for 2 days. The medium was then changed to low-glucose DMEM or endothelial cell basal medium MV2 containing $0.1 \%$ gentamicin, 1\% FBS, 20 ng/mL oncostatin M (OSM; CYT-231; ProSpec), w/ or w/o 20 ng/mL HGF (CYT244; ProSpec), and $5 \times 10^{-3} \mathrm{M}$ nicotinamide for 7 days. The medium was then changed to low-glucose DMEM containing $0.1 \%$ gentamicin, $1 \times$ insulin-transferrin-selenium (ITS) supplement $(100 \times), 20 \mathrm{ng} / \mathrm{mL}$ OSM (CYT-231; ProSpec), w/ or w/o 20 ng/mL HGF (CYT-244; ProSpec), $10 \mathrm{ng} / \mathrm{mL}$ bFGF, $1 \times 10^{-6} \mathrm{M}$ dexamethasone, $5 \times 10^{-3} \mathrm{M}$ nicotinamide, and $0.1 \%$ DMSO for an additional 14 days. The medium was replaced every $2-3$ days.

\subsection{RNA isolation and quantitative real-time polymerase chain reaction (PCR) analysis}


Total RNA was isolated using Easy-Blue reagent (Intron Biotechnology, South Korea) according to the manufacturer's protocol. Gene-specific human primers were as follows: HGF (forward: 5'-

AAGGTGACTCTGAATGAGTC-3', reverse: 5'-GGCACATCCACGACCAGGAACAATG-3'), ALB (forward: 5'GTCACCAAATGCTGCACAGA-3', reverse: 5'-ACGAGCTCAACAAGTGCAGT-3'), TDO2 (forward: 5'GTGTGCATGGTGCACAGAAT-3', reverse: 5'-GGGTTCATCTTCGGTATCCA-3'), CYP2E1(forward: 5'TTCCTCCTGCTGGTGTCCAT-3', reverse: 5'-CCCACGTACAGCGTGAACA-3') and $\beta$-actin (forward: 5'GTCCTCTCCCAAGTCCACAC-3', reverse: 5'-GGGAGACCAAAAGCCTTCAT-3'). All amplifications were conducted in a premixture $(20 \mu \mathrm{L})$ containing of $5 \mu \mathrm{M}$ gene-specific primers $4 \mu \mathrm{L}, 2 \times$ SYBR $10 \mu \mathrm{L}$, and 6 $\mu \mathrm{L}$ template, under the following conditions: denaturation at $95^{\circ} \mathrm{C}$ for $5 \mathrm{~min} ; 40$ cycles of $95^{\circ} \mathrm{C}$ for $10 \mathrm{~s}$, $59^{\circ} \mathrm{C}$ for $34 \mathrm{~s}$; and a final extension at $72{ }^{\circ} \mathrm{C}$ for $5 \mathrm{~min}$. The reactions were carried out using a Roche LC96 instrument (Roche Diagnostics, Penzberg, Germany). For validation of real-time PCR, PCR was conducted under the following conditions: denaturation at $95^{\circ} \mathrm{C}$ for $5 \mathrm{~min}$; cycles (34 for $A L B, 34$ for $T D O-2,31$ for CYP2E1, and 18 for $\beta$-actin) of $95^{\circ} \mathrm{C}$ for $30 \mathrm{~s}, 59^{\circ} \mathrm{C}$ for $30 \mathrm{~s}$, and $72{ }^{\circ} \mathrm{C}$ for $30 \mathrm{~s}$; and a final extension at $72{ }^{\circ} \mathrm{C}$ for 5 min using the same cDNA and primers. PCR products were separated on $2 \%$ agarose gels and visualized by ethidium bromide staining.

\subsection{Immunoblotting}

Cells were washed twice with ice-cold phosphate-buffered saline (PBS), lysed with an appropriate amount of tissue lysis buffer (RIPA buffer containing protease and phosphatase inhibitor cocktail), incubated on ice for $30 \mathrm{~min}$, and centrifuged at $13,000 \mathrm{rpm}$ for $10 \mathrm{~min}$ at $4{ }^{\circ} \mathrm{C}$. Next, $30 \mu \mathrm{g}$ of total protein was loaded and separated by sodium dodecyl sulfate polyacrylamide gel electrophoresis. Proteins were transferred to polyvinylidene difluoride membranes, blocked for $1 \mathrm{~h}$ with $5 \%$ nonfat dry milk in Tris-buffered saline (TBS) with $0.05 \%$ Tween-20 (TBS-T), and incubated with the appropriate primary antibodies in TBS containing $1 \%$ bovine serum albumin overnight at $4{ }^{\circ} \mathrm{C}$. Membranes were washed several times with TBST and incubated with horseradish peroxidase-conjugated secondary antibodies $(0.1 \mu \mathrm{g} / \mathrm{mL}$; Jackson ImmunoResearch Laboratories, West Grove, PA, USA). Immunoreactivity was detected using an enhanced chemiluminescence detection system (WSE 6100 LuminoGraph I; ATTO, Tokyo, Japan).

\subsection{Human Hepatocyte Growth Factor (HGF) ELISA}

To evaluate levels of HGF secretion, $200 \mu \mathrm{L}$ of conditioned media were obtained from each group. HGF ELISA was conducted using RayBio Human HGF ELISA kit (RayBiotech, ELH-HGF) according to manufacturer's instructions. HGF standards and undiluted supernatant from each group were added to the assay microplate and were allowed to incubate for 2.5 hours at room temperature. After washing, biotinylated anti-human HGF antibody and HRP-conjugated streptavidin were then applied for an hour and 45 minutes. Absorbance at $450 \mathrm{~nm}$ was read using an Epoch microplate spectrophotometer (Bio Tek Instruments).

\subsection{Immunofluorescence staining}

Cells grown or differentiated on round glass coverslips in 24-well plates were fixed and permeabilized with $100 \%$ cold methanol for 10 min. Fixed cells were incubated for $1 \mathrm{~h}$ in PBS containing $3 \%$ bovine 
serum albumin for blocking, followed by $2 \mathrm{~h}$ of incubation with specific primary antibodies. Cells were washed three times with TBS-T, then incubated with Cy2-conjugated goat anti-rabbit/mouse lgGs (Jackson Immunoresearch Laboratories) or Alexa 594-conjugated goat anti-rabbit/mouse lgGs (Molecular Probes, Eugene, OR, USA) as required according to the primary antibody. Cellular DNA was counterstained with 4',6-diamidino-2-phenylindole $(0.2 \mu \mathrm{g} / \mathrm{mL}$ in PBS).

\subsection{Periodic acid-Schiff (PAS) staining}

For PAS staining (1.01646.0001; Merck Millipore, Germany), hASCs, hESC-MSCs and their individual hepatogenic differentiated cells attached to coverslips were washed twice with PBS, fixed with $4 \%$ paraformaldehyde for $10 \mathrm{~min}$, and washed with water. The cells were incubated with $0.5 \%$ periodic acid solution for $5 \mathrm{~min}$. After rinsing with tap water, the cells were incubated with Schiff's solution for 15 min and counterstained with hematoxylin solution for $2 \mathrm{~min}$. The stained cells were observed and photographed under a microscope.

\subsection{Flow cytometry analysis}

Cells $\left(5 \times 10^{5}\right)$ were washed with PBS two times and stained with the following secondary antibodies conjugated with fluorophores: PE-CD105 (560839, BD Pharmigen), PE-CD135 (558996, BD Pharmigen) and PE-ASGRP-1 (Asialoglycoprotein receptor-1, 563655, BD Pharmigen) for $1 \mathrm{~h}$. Information on the antibody for the negative control is PE-IgG (555749, BD Pharmigen). The cells were washed with PBS two times and measured by flow cytometry on a FACS Calibur (BD Biosciences). The acquired data were analyzed using BD FACS Calibur software.

\subsection{Statistics}

Graphical data were presented as means \pm standard errors of the means (SEMs). Statistical significance among the three groups and between groups was determined using one-way or two-way analysis of variance (ANOVA) followed by Bonferroni post-tests. Differences with $p$ values of less than 0.05 were considered significant.

\section{Results}

\subsection{High expression of HGF in hESCs}

First of all, we performed real-time PCR and analyzed the expression patterns of HGF gene among mesenchymal stem cells derived from different tissues (hASCs, hBM-MSCs, hESC-MSCs) since expression level of HGF is very crucial for hepatogenic differentiation. As a result, expression of HGF was increased approximately 3.4-fold in hASCs, 7.6-fold in hESC-MSCs, compared to hBM-MSCs (Fig. 1A). Besides, western blot and ELISA analysis confirmed that the protein expression level of HGF was similar to mRNA pattern (Fig. 1B and 1C).

\subsection{Hepatogenic differentiation of hESC-MSCs}


Based on our previous study (19), we tried to differentiate hESC-MSCs into hepatocyte with or without HGF during hepatogenic differentiation. To test the potential of hESC-MSCs to differentiate into hepatocytes, we performed real-time PCR and analyzed the expression patterns of hepatocyte-specific genes, such as albumin (ALB), tryptophan 2,3-dioxygenase (TDO2) and Cytochrome P450 Family 2 Subfamily E Member 1 (CYP2E1) during the hepatogenic differentiation of hASCs and hESC-MSCs. Hepatocyte-specific genes to be identified have the following characteristics: ALB plays a critical role of formation of liver organ in early embryo (20). TD02 plays a central role in tryptophan metabolism, a major function of hepatocytes, and its expression is normally restricted to the liver (21). CYP2E1, one of monooxygenases, catalyze many reactions involved in drug metabolism and synthesis of cholesterol, steroids and other lipids (22). As shown in Fig. 2A, expression of $A L B$ was increased approximately 20fold in hASCs-derived hepatogenic differentiated cells and $30 \sim 40$-fold in hESC-MSCs-derived hepatogenic differentiated cells with or without HGF, compared to non-treated hASCs. And the expression of TDO2 was increased approximately 40-fold in hASCs-derived hepatogenic differentiated cells and 130 140-fold in hESC-MSCs-derived hepatogenic differentiated cells with or without HGF, compared to nontreated hASCs. Similarly, the expression of CYP2E1 was increased approximately 4-fold in hASCs-derived hepatogenic differentiated cells and 10-fold in hESC-MSCs-derived hepatogenic differentiated cells with or without HGF, compared to non-treated hASCs. Moreover, we confirmed that PCR bands specific for ALB, TDO-2 and CYP2E1 were denser in samples from hepatogenic-differentiated hESC-MSCs than in samples from hepatogenic-differentiated hASCs (Fig. 2B). Therefore, we concluded that even without HGF, hESCMSCs can differentiate into hepatocyte-like cells more efficiently than hASCs.

Furthermore, we performed flow cytometry to determine the expression of cell surface markers of hepatogenic-differentiated hESC-MSC cells (Fig. 3). The results showed that hESC-MSCs-derived hepatogenic differentiated cells (V1-R $9.1 \%$ of the total cells in case of hepatogenic differentiation without HGF, V1-R $10.4 \%$ of the total cells in case of hepatogenic differentiation with HGF) express less mesenchymal stem cells-specific cell surface markers such as CD105, compared to hESC-MSCs (V1-R $51.2 \%$ of the total cells). In contrast, their hepatogenic differentiated cells highly expressed cell surface markers expressed in mainly hepatocyte, such as CD135 (Flt-2) cells (V1-R 92.6\% of the total cells in case of hepatogenic differentiation without HGF, V1-R $95.3 \%$ of the total cells in case of hepatogenic differentiation with HGF)(23) and ASGRP-1 (V1-R 93.7\% of the total cells in case of hepatogenic differentiation without HGF, V1-R $94.3 \%$ of the total cells in case of hepatogenic differentiation with HGF) $(24,25)$, compared to hESC-MSCs (V1-R $54.6 \%$ of the total cells in CD135, V1-R $54.7 \%$ of the total cells in ASGRP1).

\subsection{HNF4a expression of hepatocyte-like cells differentiated from hES-MSCs}

Next, we confirmed the expression level of HNF4a (hepatocyte nuclear factor 4 alpha), a nuclear transcription factor which regulates the expression of several hepatic genes. In that, it controls the expression of hepatic genes during the transition of endodermal cells to hepatic progenitor cells, 
facilitating the recruitment of RNA pol II to the promoters of target genes (26). Therefore, considering that HNF4a was highly expressed in their hepatogenic-differentiated cells, HNF4a immunofluorescent staining was performed to test whether hASCs or hESC-MSCs were well-differentiated into hepatocyte-like cells. As shown in Fig. 4, undifferentiated cells (hASCs, hESC-MSCs) were negative for HNF4a by immunofluorescence staining, while their hepatogenic-differentiated cells were positively stained for HNF4a.

\subsection{Characteristics of hepatocyte-like cells differentiated from hES-MSCs}

Likewise, undifferentiated cells (hASCs, hESC-MSCs) were negative for ALB by immunofluorescence staining, while their hepatogenic-differentiated cells were positively stained for ALB (Fig. 5A). Moreover, their hepatogenic-differentiated cells, but not undifferentiated cells, exhibited strong PAS staining, indicating the presence of polysaccharides such as glycogen (Fig. 5B). These data suggested that hESCMSCs could be differentiated into functional hepatocyte-like cells as well as hASCs.

\section{Discussion}

Liver transplantation is the ultimate treatment for end-stage liver failure and severe acute liver failure (5, 27). However, there were many problems including shortage of liver allografts, the risk of rejection, and the high cost of the transplantation procedure and postoperative care. Recently, the following possibilities were suggested that stem-cell-based therapy might be a viable alternative therapy in patients suffered from liver diseases, considering that a number of different types of stem cells can differentiate into hepatocytes (28-30). Besides, there is known that hMSCs themselves are effective in treating liver diseases, enhancing repopulation of endogenous hepatocytes $(5,31-33)$. Actually, primary hepatocyte transplantation has rarely produced therapeutic effects because mature hepatocytes cannot be effectively expanded in vitro, and the availability of hepatocytes is often limited by shortages of donor organs $(34,35)$. Therefore, mesenchymal stem cells (MSCs) are currently considered as the most ideal cell sources for treatment of liver diseases since MSCs derived from various tissue origins appear to be therapeutically effective in the rat liver injury model, which may ultimately provide a therapeutic alternative to liver transplantation in human patients $(32,33,36)$. Among origin tissues of MSCs, human adipose-derived stem cells (hASCs) was found to have the best capacity to differentiate into hepatocytelike cells and the best therapeutic effect in treating liver diseases (5). Nevertheless, the commercialization of hASCs still has some problems including limited cell numbers and replicative lifespan, and different differentiation potential dependent on individual $(37,38)$. Recently developed hESC-MSCs have characteristics to overcome disadvantages of MSCs as well as several advantages of MSCs (safety for teratoma formation, unaltered karyotype, and multi-differentiation potential) $(16,17)$. In other words, they can generate sufficient amounts of early passage MSCs with the consistent quality. Moreover, previous studies also have shown that they have a therapeutic effect in various animal disease models $(14,16,17$, $39)$ and even could outperform primary $\operatorname{MSCs}(40,41)$. Accordingly, we would like to test how much hES- 
MSCs express HGF and investigate whether hESC-MSCs have the differentiation potential into hepatocyte-like cells. Interestingly, we achieved that expression level of HGF, a crucial factor for hepatogenic differentiation, was noticeably increased in hESC-MSCs, compared to hBM-MSCs and hASCs. Considering that HGF-overexpressing hMSCs are effective for tissue regeneration $(8,9)$, it is crucial to express endogenously high HGF. And then, we investigated whether hESC-MSCs have better hepatogenic differentiation potential than hASCs. After 4 weeks of hepatogenic differentiation, it was observed that hepatocyte specific genes including albumin, TDO-2, CYP2E1 were highly expressed in hepatocyte-like cells derived from hESC-MSCs, compared to hepatocyte-like cells derived from hASCs. Besides, we confirmed that hESC-MSCs could be better differentiated into hepatocyte through immunofluorescent staining of HNFa and PAS staining. However, we think that it would have been more ideal experimental design if more hASCs from diverse patients was used to compare the hepatocyte differentiation capacity with hESC-MSCs since differentiation capacity of hASCs varies according to the lot number and age of the fat donor.

\section{Conclusion}

Taken together, the results of this study demonstrate that hES-MSCs, highly expressing HGF, could be easily differentiated into hepatocyte-like cells even without HGF in vitro than hASCs. Therefore, we surmised that engraft with hESC-MSCs attenuated expression of inflammatory genes at the injured sites, leading to inhibition of liver cirrhosis considering that hES-MSCs could be easily differentiated into hepatocyte-like cells. Therefore, our findings show that hESC-MSCs might have promising potential as a commercial cell therapy source to treat liver failure.

\section{Declarations}

\section{Funding Statement}

This research was supported by a fund from the National Research Foundation of Korea (NRF; grant no. 2017R1A2B4002536) and Research of Korea Centers for Disease Control and Prevention (2018ER610300). This research was supported by Hallym University Research Fund (grant no.: HURF2015-39).

\section{Consent for publication}

Informed consent was obtained from all individual participants included in the study.

\section{Competing interest}

The authors declare no potential conflicts of interest.

\section{Acknowledgements}


hESC-MSCs were kindly provided by Ph.D. Eun Ju Lee (Seoul National University Hospital, Republic of Korea),

\section{Availability of data and material}

The all data used to support the findings of this study are included within the article and therefore are available from the corresponding author upon request.

\section{Authors' contributions}

JSL: Design of the research study, Data analysis, Draft of the manuscript, Interpretation

DJY: Collection and assembly of data, Performance, Data analysis

DGY: Collection and assembly of data

CHY: Collection and assembly of data

WC: Design of the research study, Interpretation

\section{Ethics declarations}

This study including all experimental procedures for human ESC-MSCs was approved by the Ethics Committee of Hangang Sacred Heart Hospital.

\section{Abbreviations}

hBM-MSCs: human bone marrow-derived mesenchymal stem cells

hASCs: human adipose tissue-derived stem cells

hESC-MSCs: human embryonic stem cell-derived mesenchymal stem cells

HGF: hepatocyte growth factor

ALB: albumin

TD02: tryptophan 2, 3-dioxygenase

CYP2E1: Cytochrome P450 Family 2 Subfamily E Member 1

HNF4a: Hepatocyte Nuclear Factor 4 Alpha

PAS staining: Periodic acid-Schiff staining 


\section{References}

1. Anghileri E, Marconi S, Pignatelli A, Cifelli P, Galié M, Sbarbati A, et al. Neuronal differentiation potential of human adipose-derived mesenchymal stem cells. Stem Cells Dev. 2008;17(5):909-16.

2. Chamberlain G, Fox J, Ashton B, Middleton J. Concise review: mesenchymal stem cells: their phenotype, differentiation capacity, immunological features, and potential for homing. Stem cells. 2007;25(11):2739-49.

3. Kim HJ, Park J-S. Usage of human mesenchymal stem cells in cell-based therapy: advantages and disadvantages. Development reproduction. 2017;21(1):1.

4. Barry FP, Murphy JM. Mesenchymal stem cells: clinical applications and biological characterization. Int J Biochem Cell Biol. 2004;36(4):568-84.

5. Manzini BM, da Silva Santos Duarte A, Sankaramanivel S, Ramos AL, Latuf-Filho P, Escanhoela C, et al. Useful properties of undifferentiated mesenchymal stromal cells and adipose tissue as the source in liver-regenerative therapy studied in an animal model of severe acute fulminant hepatitis. Cytotherapy. 2015;17(8):1052-65.

6. Lee JS, Lee MO, Moon BH, Shim SH, Fornace AJ Jr, Cha HJ. Senescent growth arrest in mesenchymal stem cells is bypassed by Wip1-mediated downregulation of intrinsic stress signaling pathways. Stem cells. 2009;27(8):1963-75.

7. Zhao L, Liu X, Zhang Y, Liang X, Ding Y, Xu Y, et al. Enhanced cell survival and paracrine effects of mesenchymal stem cells overexpressing hepatocyte growth factor promote cardioprotection in myocardial infarction. Experimental cell research. 2016;344(1):30-9.

8. Song YS, Lee HJ, Doo SH, Lee SJ, Lim I, Chang K-T, et al. Mesenchymal stem cells overexpressing hepatocyte growth factor (HGF) inhibit collagen deposit and improve bladder function in rat model of bladder outlet obstruction. Cell Transplant. 2012;21(8):1641-50.

9. Chen S, Chen X, Wu X, Wei S, Han W, Lin J, et al. Hepatocyte growth factor-modified mesenchymal stem cells improve ischemia/reperfusion-induced acute lung injury in rats. Gene therapy. 2017;24(1):3-11.

10. Liu Y. Hepatocyte growth factor in kidney fibrosis: therapeutic potential and mechanisms of action. American Journal of Physiology-Renal Physiology. 2004;287(1):F7-16.

11. Matsuda Y, Matsumoto K, Yamada A, Ichida T, Asakura H, Komoriya $Y$, et al. Preventive and therapeutic effects in rats of hepatocyte growth factor infusion on liver fibrosis/cirrhosis. Hepatology. 1997;26(1):81-9.

12. Xia J-L, Dai C, Michalopoulos GK, Liu Y. Hepatocyte growth factor attenuates liver fibrosis induced by bile duct ligation. Am J Pathol. 2006;168(5):1500-12.

13. Mizuno S, Matsumoto K, Li M-Y, Nakamura T. HGF reduces advancing lung fibrosis in mice: a potential role for MMP-dependent myofibroblast apoptosis. The FASEB journal. 2005;19(6):580-2.

14. Lee EJ, Xu L, Kim G-H, Kang SK, Lee S-W, Park S-H, et al. Regeneration of peripheral nerves by transplanted sphere of human mesenchymal stem cells derived from embryonic stem cells. 
Biomaterials. 2012;33(29):7039-46.

15. Yoon D, Yoon D, Sim H, Hwang I, Lee J-S, Chun W. Accelerated Wound Healing by Fibroblasts Differentiated from Human Embryonic Stem Cell-Derived Mesenchymal Stem Cells in a Pressure Ulcer Animal Model. Stem Cells International. 2018;2018.

16. Lee EJ, Lee H-N, Kang H-J, Kim K-H, Hur J, Cho H-J, et al. Novel embryoid body-based method to derive mesenchymal stem cells from human embryonic stem cells. Tissue Eng Part A. 2010;16(2):705-15.

17. Hwang NS, Varghese S, Lee HJ, Zhang Z, Ye Z, Bae J, et al. In vivo commitment and functional tissue regeneration using human embryonic stem cell-derived mesenchymal cells. Proceedings of the National Academy of Sciences. 2008;105(52):20641-6.

18. Ninagawa N, Murakami R, Isobe E, Tanaka Y, Nakagawa H, Torihashi S. Mesenchymal stem cells originating from ES cells show high telomerase activity and therapeutic benefits. Differentiation. 2011;82(3):153-64.

19. Lee J-S, Yoon H, Yoon D, Kim GH, Yang HT, Chun W. Development of hepatic blocks using human adipose tissue-derived stem cells through three-dimensional cell printing techniques. Journal of Materials Chemistry B. 2017;5(5):1098-107.

20. Cascio S, Zaret KS. Hepatocyte differentiation initiates during endodermal-mesenchymal interactions prior to liver formation. Development. 1991;113(1):217-25.

21. Rodrigues ML, Pereira IA, Archer M. The Nrf H Cytochrome c Quinol Dehydrogenase. Handbook of Metalloproteins. 2006.

22. Fer M, Corcos L, Dreano Y, Plee-Gautier E, Salaun JP, Berthou F, et al. Cytochromes P450 from family 4 are the main omega hydroxylating enzymes in humans: CYP4F3B is the prominent player in PUFA metabolism. J Lipid Res. 2008;49(11):2379-89.

23. Hong SH, Gang EJ, Jeong JA, Ahn C, Hwang SH, Yang IH, et al. In vitro differentiation of human umbilical cord blood-derived mesenchymal stem cells into hepatocyte-like cells. Biochem Biophys Res Commun. 2005;330(4):1153-61.

24. Peters DT, Henderson CA, Warren CR, Friesen M, Xia F, Becker CE, et al. Asialoglycoprotein receptor 1 is a specific cell-surface marker for isolating hepatocytes derived from human pluripotent stem cells. Development. 2016;143(9):1475-81.

25. Mallanna SK, Cayo MA, Twaroski K, Gundry RL, Duncan SA. Mapping the cell-surface Nglycoproteome of human hepatocytes reveals markers for selecting a homogeneous population of iPSC-derived hepatocytes. Stem cell reports. 2016;7(3):543-56.

26. DeLaForest A, Di Furio F, Jing R, Ludwig-Kubinski A, Twaroski K, Urick A, et al. HNF4A Regulates the Formation of Hepatic Progenitor Cells from Human iPSC-Derived Endoderm by Facilitating Efficient Recruitment of RNA Pol II. Genes (Basel). 2018;10(1).

27. Khan SA, Shah N, Williams R, Jalan R. Acute liver failure: a review. Clinics in liver disease. 2006;10(2):239-58. 
28. Lee KD, Kuo TKC, Whang-Peng J, Chung YF, Lin CT, Chou SH, et al. In vitro hepatic differentiation of human mesenchymal stem cells. Hepatology. 2004;40(6):1275-84.

29. Sgodda M, Aurich H, Kleist S, Aurich I, König S, Dollinger MM, et al. Hepatocyte differentiation of mesenchymal stem cells from rat peritoneal adipose tissue in vitro and in vivo. Experimental cell research. 2007;313(13):2875-86.

30. Seo MJ, Suh SY, Bae YC, Jung JS. Differentiation of human adipose stromal cells into hepatic lineage in vitro and in vivo. Biochem Biophys Res Commun. 2005;328(1):258-64.

31. Lee MJ, Jung J, Na KH, Moon JS, Lee HJ, Kim JH, et al. Anti-fibrotic effect of chorionic plate-derived mesenchymal stem cells isolated from human placenta in a rat model of CCl4-injured liver: Potential application to the treatment of hepatic diseases. Journal of cellular biochemistry. 2010;111(6):1453-63.

32. Kuo TK, Hung SP, Chuang CH, Chen CT, Shih YRV, Fang SCY, et al. Stem cell therapy for liver disease: parameters governing the success of using bone marrow mesenchymal stem cells. Gastroenterology. 2008;134(7):2111-21. e3.

33. Forbes SJ, Newsome PN. New horizons for stem cell therapy in liver disease. Journal of hepatology. 2012;56(2):496-9.

34. Lazaro CA, Croager EJ, Mitchell C, Campbell JS, Yu C, Foraker J, et al. Establishment, characterization, and long-term maintenance of cultures of human fetal hepatocytes. Hepatology. 2003;38(5):1095-106.

35. Lloyd TD, Orr S, Skett P, Berry DP, Dennison AR. Cryopreservation of hepatocytes: a review of current methods for banking. Cell tissue banking. 2003;4(1):3-15.

36. Takami T, Terai S, Sakaida I. Stem cell therapy in chronic liver disease. Current opinion in gastroenterology. 2012;28(3):203-8.

37. Kassem M, Abdallah BM, Yu Z, Ditzel N, Burns JS. The use of hTERT-immortalized cells in tissue engineering. Cytotechnology. 2004;45(1-2):39-46.

38. Truong NC, Bui KH-T, Van Pham P, editors. Characterization of senescence of human adipose-derived stem cells after long-term expansion. Tissue Engineering and Regenerative Medicine; 2019: Springer.

39. Thiel A, Yavanian G, Nastke M-D, Morales P, Kouris NA, Kimbrel EA, et al. Human embryonic stem cellderived mesenchymal cells preserve kidney function and extend lifespan in NZB/W F1 mouse model of lupus nephritis. Scientific reports. 2015;5:17685.

40. Wang X, Kimbrel EA, ljichi K, Paul D, Lazorchak AS, Chu J, et al. Human ESC-derived MSCs outperform bone marrow MSCs in the treatment of an EAE model of multiple sclerosis. Stem cell reports. 2014;3(1):115-30.

41. Hawkins KE, Corcelli M, Dowding K, Ranzoni AM, Vlahova F, Hau KL, et al. Embryonic stem cellderived mesenchymal stem cells (MSCs) have a superior neuroprotective capacity over fetal MSCs in the hypoxic-ischemic mouse brain. Stem cells translational medicine. 2018;7(5):439-49.

\section{Figures}


Figure 1

A

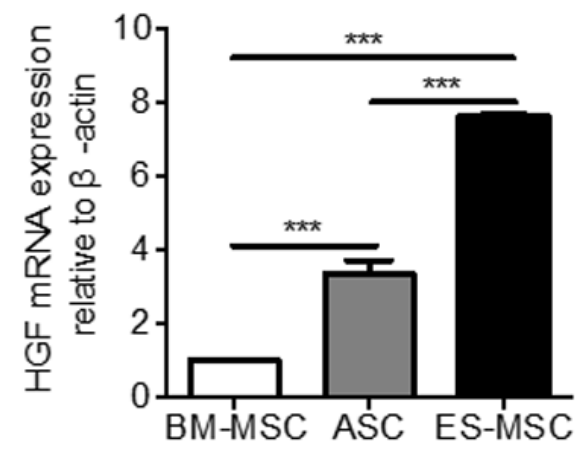

B

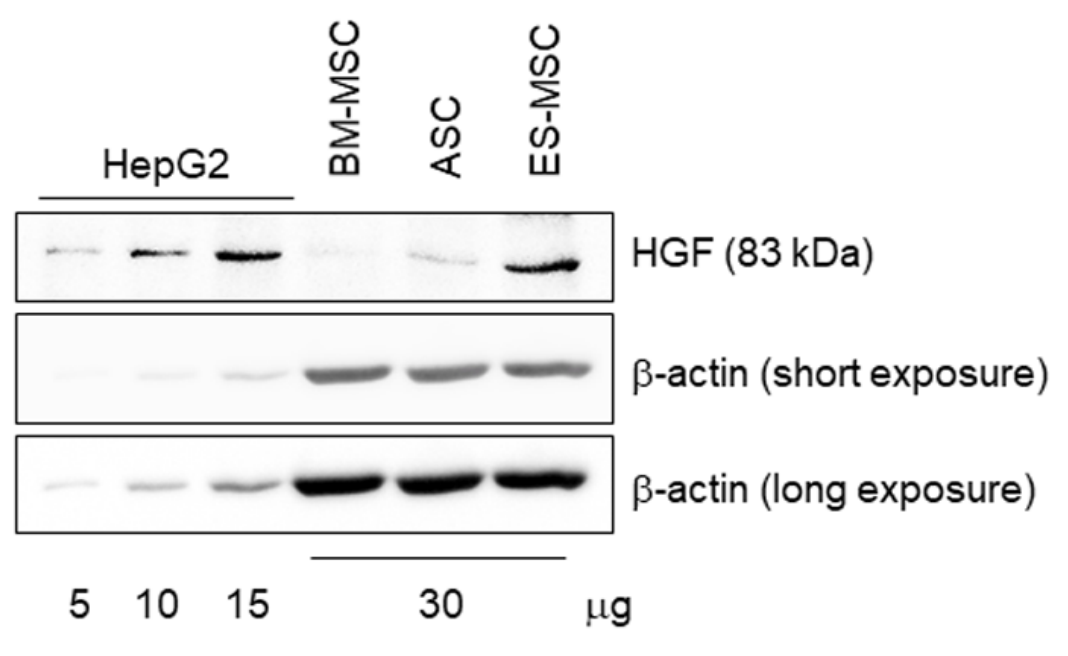

C

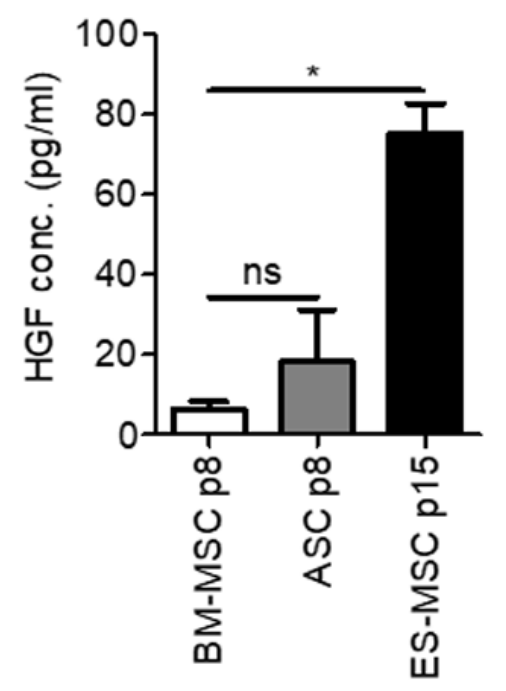

Figure 1

HGF expression level in hESC-MSCs (A) The mRNA level of HGF in bone marrow-, adipose- and embryonic stem cells-derived hMSCs was determined by real-time PCR. $\beta$-actin was used as an internal control $(n=$ 3 , one-way ANOVA; $* \star \star x p<0.001$ ). (B) HGF levels were determined via immunoblotting analysis. $\beta$-actin was employed as a protein loading control. HepG2 cells were used as a positive control of HGF 
immunoblotting band. (C) The amount of HGF secreted into conditioned media was detected by HGF ELISA assay.

Figure 2

A
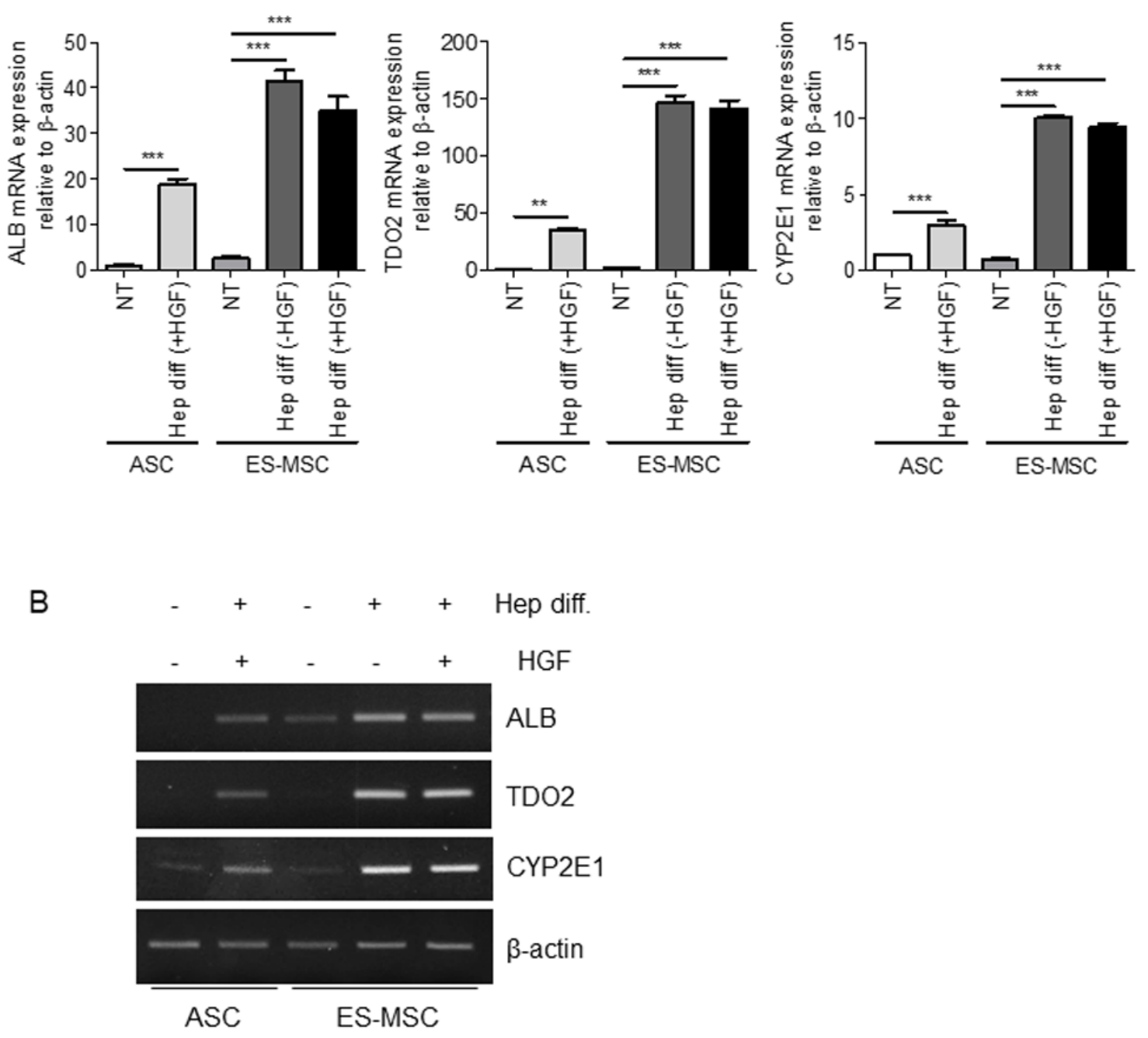

\section{Figure 2}

Hepatogenic differentiation of hESC-MSCs in vitro Human ASCs and ESC-MSCs were differentiated under hepatogenic differentiation conditions for 25 days. (A) ALB, TDO-2 and CYP2E1 mRNA levels were determined by real-time PCR after culture under hepatogenic differentiation conditions compared with 
those in untreated cells. $\beta$-actin was used as an internal control $\left(n=3\right.$, one-way ANOVA; ${ }^{\star \star} p<0.01$, $* \star \star p<$ 0.001). (B) For validation of real-time PCR, PCR was conducted and agarose gel images were shown.

\section{Figure 3}
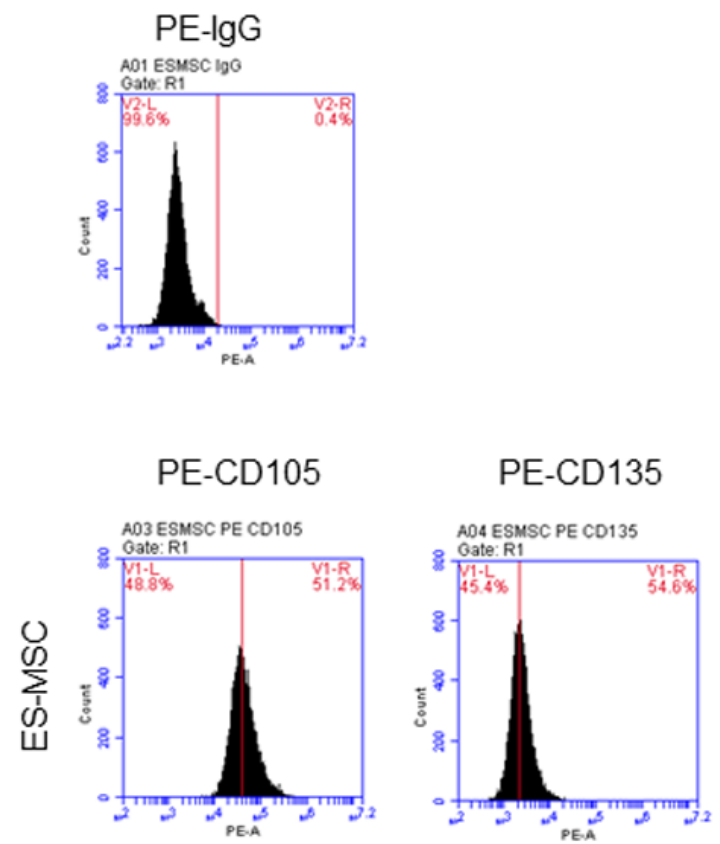

PE-ASGRP-1
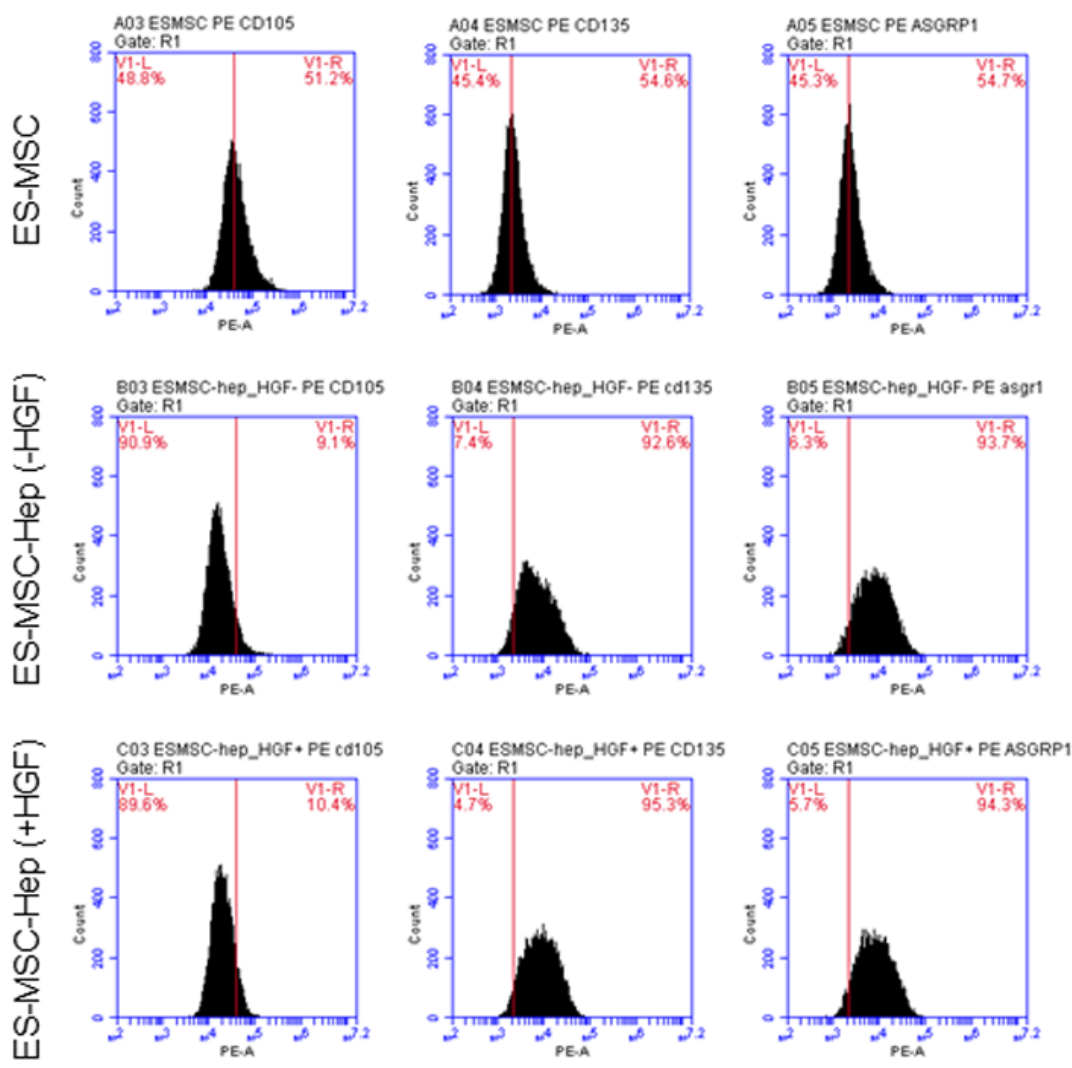

\section{Figure 3}

Flow cytometry analysis of hESC-MSC-driven hepatogenic differentiated cells in vitro Human ESC-MSCs were differentiated under hepatogenic differentiation conditions for 21 days. hESC-MSCs and their 
hepatogenic differentiated cells were trypsinized, stained with specific markers (CD105, CD135 and ASGRP-1) and measured by flow cytometry.

Figure 4

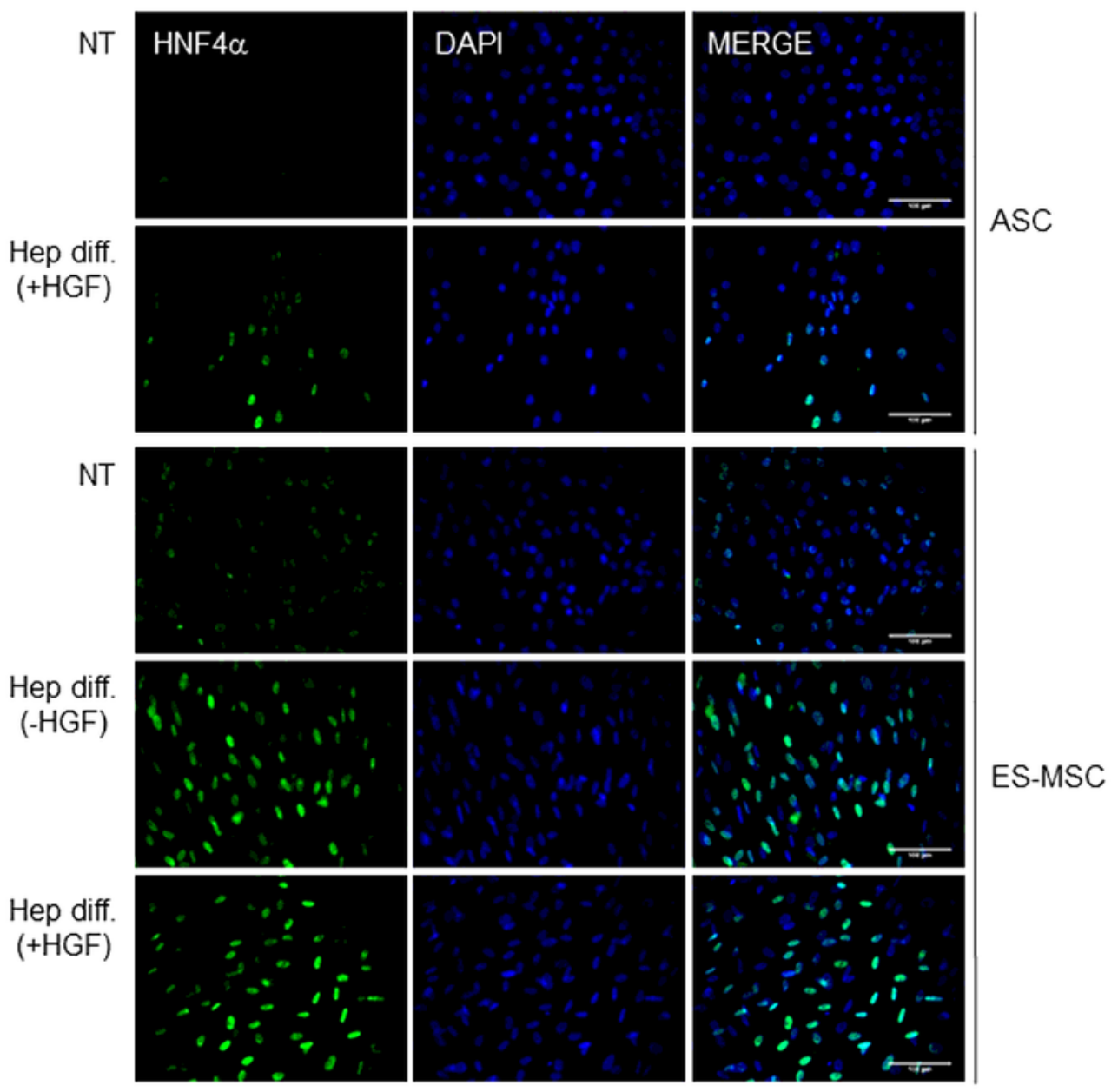

Figure 4

Immunofluorescent staining of HNF4a hASCs, hESC-MSCs and their individual hepatogenic differentiated cells were immunostained to detect HNF4a. 4',6'-Diamidino-2-phenylindole (DAPI) was used for nuclear counterstaining. 
Figure 5

A

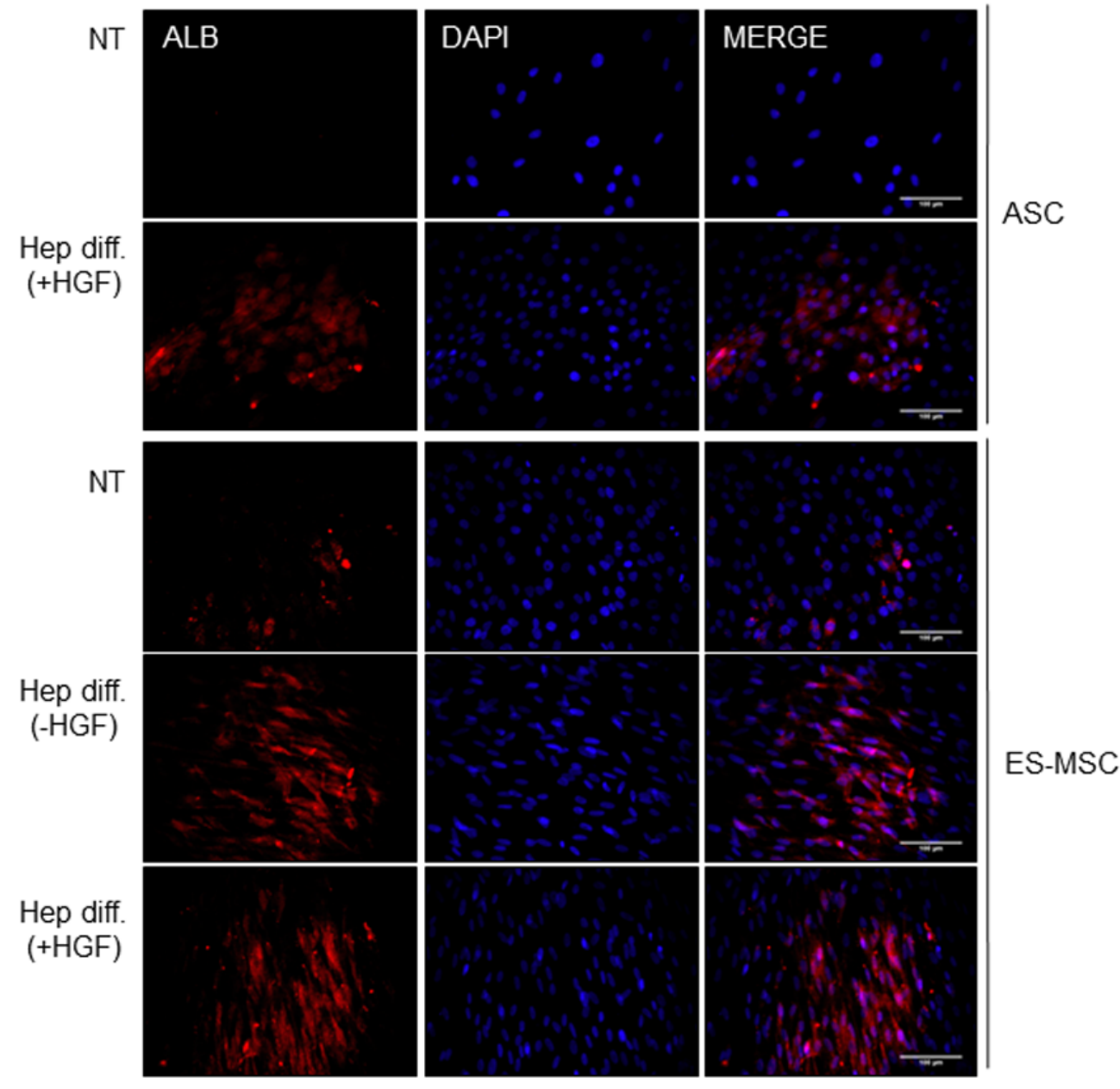

B

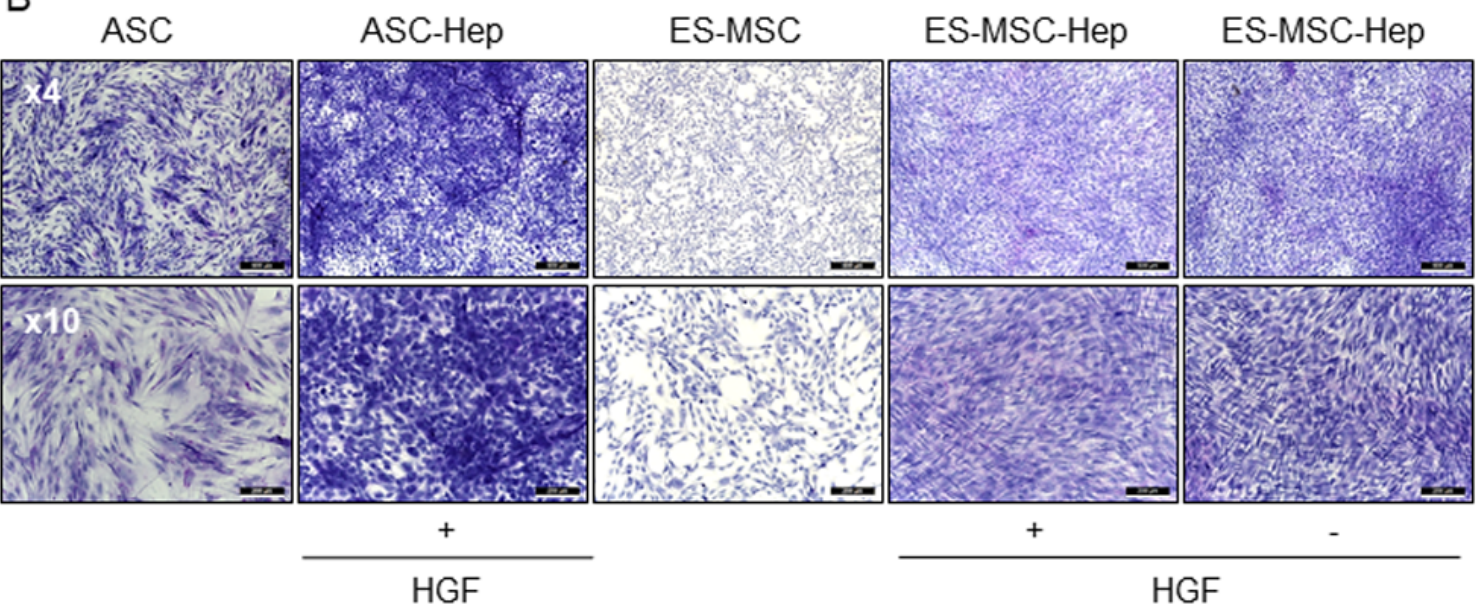

Figure 5

Characteristics of hepatocyte-like cells differentiated from hES-MSCs (A) hASCs, hESC-MSCs and their individual hepatogenic differentiated cells were immunostained to detect ALB. DAPI was used for nuclear counterstaining. (B) PAS staining for detection of glycogen storage was performed in hASCs, hESC-MSCs and their individual hepatogenic differentiated cells. 


\section{Supplementary Files}

This is a list of supplementary files associated with this preprint. Click to download.

- SupplementaryFigure1.TIF 